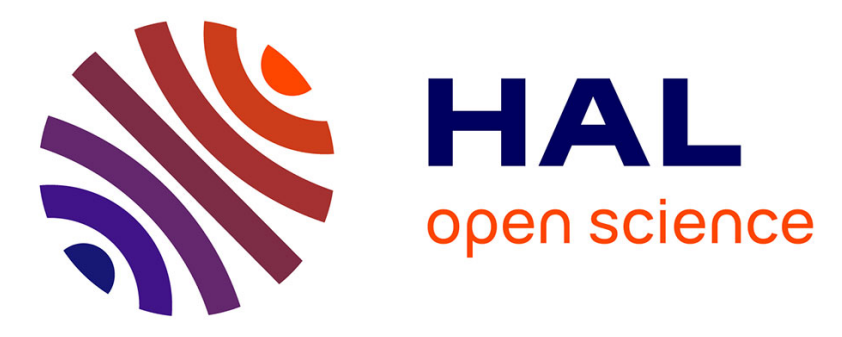

\title{
Migration and deformation of leukocytes in pressure driven flows
}

\author{
Quan Jin, Claude Verdier, Pushpendra Singh, Nadine Aubry, Roxana \\ Chotard-Ghodsnia, Alain Duperray
}

\section{- To cite this version:}

Quan Jin, Claude Verdier, Pushpendra Singh, Nadine Aubry, Roxana Chotard-Ghodsnia, et al.. Migration and deformation of leukocytes in pressure driven flows. Mechanics Research Communications, 2007, 34 (5-6), pp.411-422. 10.1016/j.mechrescom.2007.07.002 . hal-00196755

\section{HAL Id: hal-00196755 \\ https://hal.science/hal-00196755}

Submitted on 13 Dec 2007

HAL is a multi-disciplinary open access archive for the deposit and dissemination of scientific research documents, whether they are published or not. The documents may come from teaching and research institutions in France or abroad, or from public or private research centers.
L'archive ouverte pluridisciplinaire HAL, est destinée au dépôt et à la diffusion de documents scientifiques de niveau recherche, publiés ou non, émanant des établissements d'enseignement et de recherche français ou étrangers, des laboratoires publics ou privés. 


\title{
Migration and deformation of leukocytes in pressure driven flows
}

\author{
Quan Jin, Claude Verdier ${ }^{+}$, Pushpendra Singh, Nadine Aubry ${ }^{++}$, \\ Roxana Chotard-Ghodsnia ${ }^{+}$and Alain Duperray* \\ New Jersey Institute of Technology, Department of Mechanical Engineering, Newark, New Jersey 07102, USA \\ ${ }^{+}$Laboratoire de Spectrométrie Physique, CNRS, UMR 5588, Université Joseph Fourier, BP87, 140 avenue de la \\ Physique, 38402 Saint Martin d'Hères cedex, France \\ ${ }^{++}$Department of Mechanical Engineering, Carnegie Mellon University, Pittsburgh, PA 15213, USA \\ *INSERM, U578, Grenoble, France, Université Grenoble I, Groupe de Recherche sur le Cancer du Poumon, \\ Institut Albert Bonniot, Grenoble, France
}

\begin{abstract}
Direct numerical simulations (DNS) are used to study the motion and deformation of leukocytes in pressure driven flows in parallel plate channels. The influence of the adhesion force between the leukocytes and the channel wall on such motion and deformation is also investigated. Leukocytes are represented by two composite fluid models, consisting of a membrane, a cytoplasm and a nucleus. The adhesion force is computed using two adhesion force models. In the first model, the adhesion force is given by a potential, and in the second model it is given by Dembo's kinetic adhesion model. The numerical code is based on the finite element method and the level-set technique is used to track the cell membrane position. In the absence of the adhesion force, the leukocyte moves away from the wall to an equilibrium location that depends on the ratio of the cell to plasma viscosities. In presence of the adhesion force, the leukocyte is attracted to the layer of endothelial cells and, as it gets closer, it also flattens under the action of hydrodynamic forces. This deformation, in turn, further increases the adhesion force. The leukocyte, however, can be captured only when it is placed sufficiently close to the wall, which for the kinetic model is of the order of $30 \mathrm{~nm}$. We also find that for the normal parameter values and flow rates the adhesive force given by the kinetic model is too small to capture the leukocyte.
\end{abstract}

\section{INTRODUCTION}

Although the white blood cells (WBC or leukocytes) constitute only a small fraction of blood cells, they play a very important role in the body's immune response. They do so either by fighting pathogens, viruses, or other cells (cancer cells for example), or by reaching the site of infection through the transendothelial migration. The endothelial monolayer is a part of the vessel wall. Endothelial cells are maintained in close contact with each other by a complex network of transmembrane adhesion proteins, especially cadherins. Diapedesis is the way leukocytes migrate through the endothelial junctions under the combined effects of signalisation, changes in the rheological properties and the cell-cell adhesion properties. As part of the migration process, circulating leukocytes must first adhere to the luminal surface of the endothelium (see figure 1).

The goal of this paper focuses on the first phase of the above process, i.e., the study of the motion and deformation of leukocytes in small vessels and the investigation of conditions under which leukocytes can remain attached to the vessel wall and do not drift away. For this, we will assume that the leukocyte is already close to the endothelium. The factors, that cause leukocytes to get close to the endothelium in the first place, such as collisions with other cells, will not be studied. This work should also be helpful in improving our understanding of the motion of cancerous cells, and the role of the flow rate and the cortical tension in the process [1]. Indeed, it has been observed that the mechanisms by which cancer cells [1-3] migrate to and through the endothelium to reach different tissues are similar to the ones used by leukocytes, although the adhesion molecules involved for the former are not all known yet [4].

Past recent numerical studies [5-7], based on a compound-drop model, have been capable of qualitatively explaining the behavior of leukocytes observed in experiments. The compound-drop model of a leukocyte consists of a spherical nucleus containing a core fluid which is surrounded by a thick layer 
of cytoplasm. In this paper, we use both this compound-drop model and a drop-rigid-particle model to represent leukocytes. The two most common adhesion models are based on Bell's equilibrium model and Dembo's kinetics model [8-11], which are both used here to explore the adhesion mechanisms.

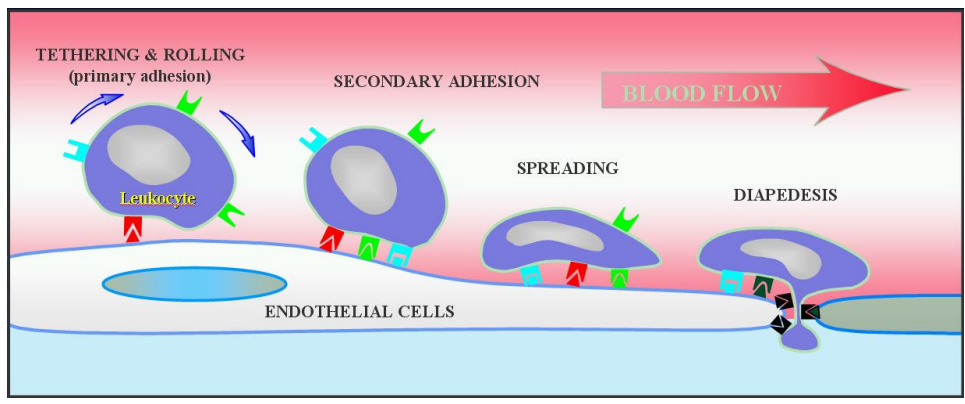

Figure 1. The different steps corresponding to the leukocyte extravasation.

\section{MODELING AND GOVERNING EQUATIONS}

\subsection{Rheological models for leukocytes}

In this study leukocytes, which are the only blood cells with a nucleus, will be represented using two different two-layered models in which the outer layer represents the cytoplasm and the inner layer represents the nucleus [5,7]. The two-layered models of leukocytes used here approximately represent neutrophils, although for simplicity the shape of the nucleus is assumed to be spherical.

In the first two-layered model shown in Figure 2a, called the drop-rigid-particle model, the cytoplasm is assumed to be a Newtonian fluid, and the inner core is assumed to be rigid and thus modeled as a rigid particle. The nucleus volume is assumed to be $21 \%$ of the total cell volume, and thus the thickness of the outer shell in this model is $2.7 \mu \mathrm{m}$. The surface or cortical tension coefficient for the interface between the cytoplasm and the surrounding fluid (plasma) is assumed to be $3.0 \times 10^{-5} \mathrm{~N} / \mathrm{m}$. The viscosity of the cytoplasm is higher than that of the plasma. The cell radius is assumed to be $6.5 \mu \mathrm{m}$, as in [12], which is larger than the commonly accepted mean radius of a neutrophil that is around $4.3 \mu \mathrm{m}$ [13].

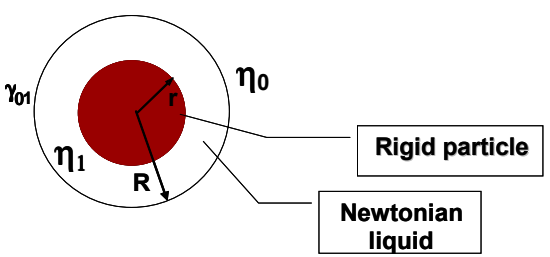

(a)

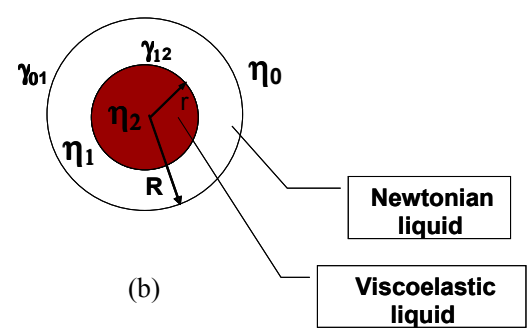

Figure 2. Two layered models of leukocytes. (a) drop-rigid-particle model, (b) compound-drop model.

In the second model shown in Figure $2 b$, called the compound-drop model, the outer layer is the same as above but the nucleus is modeled by a viscoelastic liquid. Thus, in addition to the outer interface between the shell and the surrounding fluid, there is an interface between the core fluid and the shell. The surface tension for both interfaces is assumed to be $3.0 \times 10^{-5} \mathrm{~N} / \mathrm{m}$ and the viscosity of the viscoelastic liquid inside the nucleus is assumed to be higher than that of the shell. The values of the parameters for the two previous models are given in Table 1 . The densities of the plasma and leukocyte are assumed to be equal, and thus the gravity plays no role in the dynamics.

\subsection{Adhesion models}

Under normal conditions, leukocytes are freely suspended in the blood stream. However, upon inflammation, the leukocytes that come close to the endothelial layer, as a result of the collisions with other blood cells and the expression or activation of adhesion molecules, can be captured. This latter process can be modeled by an adhesion force $\mathbf{F}_{\mathbf{b}}$, which acts between the leukocytes and the endothelium. 
This force has a very short range and can result in the capture of leukocytes that come sufficiently close to the wall. We next describe two models that are used to represent the adhesion force.

\begin{tabular}{|c|c|}
\hline Parameters & Values used \\
\hline Radius, $\mathrm{R}$ & $6.5 \mu \mathrm{m}[14,7]$ \\
\hline Shell Thickness, R-r & $2.7 \mu \mathrm{m}[5]$ \\
\hline Cortical tension, $\gamma_{10}$ & $3 \times 10^{-5} \mathrm{~N} / \mathrm{m}$ (Ref. [15]) \\
\hline Interfacial tension, $\gamma_{12}$ & $3 \times 10^{-5} \mathrm{~N} / \mathrm{m}$ (Ref. [15] \\
\hline Density, $\rho$ & $1000 \mathrm{~kg} / \mathrm{m}^{3}$ (Ref. [5]) \\
\hline Viscosity of cytoplasm, $\eta_{1}$ & 35.28 Pa.s (Ref. [16]) \\
\hline Plasma viscosity, $\eta_{0}$ & 0.001 Pa.s (Ref. [14]) \\
\hline Viscosity ratio $\eta_{0} / \eta_{1}$ & $0.01[17]$ \\
\hline *Viscosity ratio $\eta_{1} / \eta_{2}$ & $0.35[17,16]$ \\
\hline *Relaxation time of nucleus, $\lambda_{\mathrm{r}}$ & $0.1 \mathrm{~s}[18]$ \\
\hline *Viscosity of nucleus, $\eta_{2}$ & 100.0 Pa.s [17,16] \\
\hline
\end{tabular}

Table 1. Parameters for the two-layered leukocyte models. Parameters marked with a star apply to the compounddrop model only. Appropriate references for the choice of these values have been included in brackets.

\subsection{Adhesion potential model}

In the first model, introduced by Sukumaran and Seifert [11], the adhesion potential of the leukocyte with a plane wall at $x=0$ is assumed to be

$$
W(x)=w\left(\frac{d_{0}}{x}\right)^{2}\left[\left(\frac{d_{0}}{x}\right)^{2}-2\right]
$$

where $w$ is the adhesion strength and $d_{0}=0.02 R$ is a constant related to the cell radius $R$. Depending on its magnitude, the latter models both specific and nonspecific interactions.

\subsection{Kinetic adhesion model}

The second adhesion model is the kinetic model of Dembo et al. [9]. Here, the adhesion force between the cell membrane and the wall is assumed to arise due to the formation of bonds between the specific adhesion molecules on the cell membrane and the receptors on the substrate. A bond between the cell and the substrate is modeled as a Hookean spring, and the corresponding force $f_{b}$ due to one bond is

$$
f_{b}=\sigma\left(x_{m}-l_{m v}-\lambda\right)
$$

where $\sigma$ is the spring constant, $x_{m}$ is the distance between the cell surface and the wall, $l_{m v}$ is the unstressed microvillus length [7,19-21], and $\lambda$ is the equilibrium or unstretched bond length. The total force per unit surface area $F_{b}$ between the cell and the substrate is then deduced from

$$
F_{b}=N_{b} f_{b}
$$

where $N_{b}$ is the bond density per unit area. The time evolution of the latter is given by a kinetic equation which balances the formation and dissociation of bonds:

$$
\frac{\partial N_{b}}{\partial t}=k_{f}\left(N_{l 0}-N_{b}\right)\left(N_{r 0}-N_{b}\right)-k_{r} N_{b},
$$

where $N_{l 0}$ and $N_{r 0}$ are the initial ligands and receptors densities on the surface of the cell membrane.

The reverse and forward reaction rate coefficients $k_{r}$ and $k_{f}$ in (4) are given by:

$$
\begin{gathered}
k_{r}=k_{r 0} \exp \left(\frac{\left(\sigma-\sigma_{t s}\right)\left(x_{m}-l_{m v}-\lambda\right)^{2}}{2 k_{b} T}\right) \\
k_{f}=k_{f 0} \exp \left(-\frac{\sigma_{t s}\left(x_{m}-l_{n v}-\lambda\right)^{2}}{2 k_{b} T}\right)
\end{gathered}
$$


where $k_{r 0}$ and $k_{f 0}$ are the equilibrium values of the reverse and forward reaction rates, $\sigma$ and $\sigma_{\mathrm{ts}}$ are the spring and transition spring constants, $k_{b}$ is the Boltzmann constant, and $T$ is the temperature. The values of these parameters are listed in Table 2.

The equilibrium bond density $N_{b 0}$ reaches the maximum value $N_{b 0 m}$ when the bond stretching $x=x_{m}$ $\lambda-l_{m v}$ is zero. For a given value of $x_{m}$, the equilibrium bond density $N_{b 0}$ satisfies the following quadratic equation, which is deduced by setting the time dependent term in (4) equal to zero

$$
k_{f}\left(N_{l 0}-N_{b 0}\right)\left(N_{r 0}-N_{b 0}\right)-k_{r} N_{b 0}=0
$$

and which admits two solutions:

$$
N_{1,2}=\frac{\left(N_{r 0}+N_{l 0}+\frac{k_{r}}{k_{f}}\right) \pm D}{2}
$$

where $\mathrm{D}$ is the discriminant of the quadratic equation, i.e.

$$
D=\sqrt{\left(N_{r 0}+N_{l 0}+\frac{k_{r}}{k_{f}}\right)^{2}-4 N_{r 0} N_{l 0}}
$$

The larger solution $N_{1}$ leading to a value larger than the number of available bonds is not physically meaningful. The only physical solution of (7) is thus $N_{2}$, leading to the equilibrium bond density:

$$
N_{b 0}=\frac{\left(N_{r 0}+N_{l 0}+\frac{k_{r}}{k_{f}}\right)-D}{2}
$$

For the parameter values listed in Table 2, the maximum value of the equilibrium bond density, which is deduced by setting $k_{f}=k_{f 0}$ and $k_{r}=k_{r 0}$, is $N_{b 0 m}=1.5 \times 10^{14} \mathrm{~m}^{-2}$.

\begin{tabular}{|l|c|c|}
\hline \multicolumn{1}{|c|}{ Parameters } & Range & $\begin{array}{c}\text { Values used in } \\
\text { calculations }\end{array}$ \\
\hline Initial ligand density on the surface, $N_{t 0}, m^{-2}$ & $2.0-5.0 \times 10^{14}[9]$ & $1.5 \times 10^{14}$ \\
\hline Initial receptors density on the cell membrane, $N_{r 0}, m^{-2}$ & $2.0-5.0 \times 10^{14}[9]$ & $3.0 \times 10^{14}$ \\
\hline Initial reverse reaction rate, $k_{r 0}, 1 / s$ & $10^{-5}-10[8]$ & 10 \\
\hline Initial forward reaction rate, $k_{f 0}, \mathrm{~m}^{2} / \mathrm{s}$ & $10^{-18}-10^{-10}[8]$ & $10^{-10}$ \\
\hline Spring constant, $\sigma, \mathrm{N} / \mathrm{m}$ & $0.00001-0.01[8]$ & $2 \times 10^{-4}$ \\
\hline Transition spring constant, $\sigma_{t s}, \mathrm{~N} / \mathrm{m}$ & $-0.005-0.0095[8]$ & $10^{-4}$ \\
\hline Unstressed microvillus length, $l_{m v}, \mathrm{~m}$ & $3 \times 10^{-7}[19,21]$ & $3 \times 10^{-7}$ \\
\hline Equilibrium bond length, $\lambda, \mathrm{m}$ & $10^{-8} \sim 10^{-7}[19]$ & $10^{-8}$ \\
\hline Thermal energy, $k_{b} T, N \cdot m$ & $3.8 \sim 4.3 \times 10^{-21}$ & $4.28 \times 10^{-21}$ \\
\hline
\end{tabular}

Table 2. Parameter values for the kinetic adhesion model. Appropriate references have been included in brackets.

Both the equilibrium bond density $N_{b 0}$ and the adhesion force vary with the distance between the cell membrane and the wall. In Figure 3a the adhesion force and bond density are shown as a function of the dimensionless bond stretching $x^{\prime}=\frac{x}{\lambda+l_{m v}} \cdot 10^{3}$. Notice that when $x^{\prime}$ is approximately 48 the force reaches the maximum possible value. In terms of the dimensional variables, the force is maximum when $\left|x_{m}-\lambda-l_{m v}\right|=14.9 \mathrm{~nm}$, and the adhesion force is approximately zero when $\left|x_{m}-\lambda-l_{m v}\right|$ is greater than $27.9 \mathrm{~nm}$. Therefore, the force is present only over the narrow distance range of $\sim 30 \mathrm{~nm}$. Consequently, as discussed later, a cell can be captured only if it is located within the above range.

We next consider the time evolution of $N_{b}$ when $x_{m}$ is suddenly increased from $\left(\lambda+l_{m v}\right)$ at $\mathrm{t}=0$, to $1.01\left(\lambda+l_{m v}\right)$, which causes the bonds to break and the bond density to evolve to a new equilibrium value (see Figure $3 \mathrm{~b}$ ). The bond density $N_{b}$ in this case decays with time from $N_{b 0 m}$ to a smaller value. Our 
objective here is to determine the characteristic time over which the bond density evolves. This is important because in a flow situation the distance between the cell wall and the endothelial monolayer changes with time. Clearly, if this characteristic time over which the new equilibrium value is reached is much smaller than the flow time scale, it is appropriate to assume in the fluid dynamics problem that the bond density is given by the steady solution of equation (4).

(a)

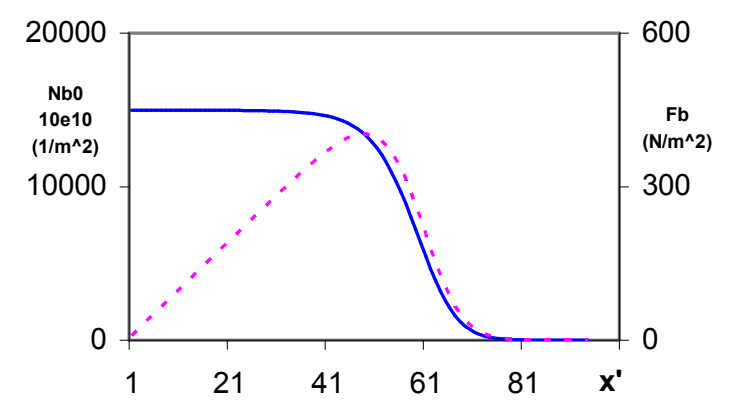
functions of the dimensionless bond stretching x'. (b) The bond density as a function of time when at $\mathrm{t}=0, x_{m}$ is suddenly increased from $\left(\lambda+l_{m v}\right)$ to $1.01\left(\lambda+l_{m v}\right)$. The new equilibrium value is approximately reached at $\mathrm{t}=7 \times 10^{-6} \mathrm{~s}$.

By integrating equation (4) analytically, it is easy to show that

$$
N(t)=\frac{N_{2}-\beta N_{1} \exp (-t / \tau)}{1-\beta \exp (-t / \tau)}
$$

where $N_{1}$ and $N_{2}$ are the two roots defined in (8) and $D$ is given by (9), $\tau=\frac{1}{k_{f} D}, \beta=\frac{\alpha-N_{2}}{\alpha-N_{1}}<0$, and $\alpha$ is the initial bond density which, for the case considered, is $N_{b 0 m}$.

From equation (11) it is clear that as $t \rightarrow \infty$, we obtain $N(t)=N_{2}$ as the solution for the new equilibrium state. Furthermore, it is interesting to notice that for all $t>0$, we have

$$
N^{\prime}(t)=\frac{\left(N_{1}-N_{2}\right) \beta \exp (-t / \tau)}{\tau(1-\beta \exp (-t / \tau))^{2}}<0 .
$$

We then define the characteristic time $t_{c}$ by setting $N_{2}=N^{\prime}(0) t_{\mathrm{c}}+\alpha$, which after using (12) gives,

$$
t_{c}=\frac{\tau\left(N_{1}-N_{2}\right)}{\left(N_{1}-\alpha\right)}=\frac{1}{k_{f}\left(N_{1}-\alpha\right)}
$$

From the above expression, we deduce that while $t_{c}$ depends on $k_{f}, k_{r}, N_{r 0}, N_{l 0}$, it is independent of $\sigma_{t s}, \sigma$.

For the parameter values listed in Table 2 , the characteristic time $t_{c}$ given by equation (13) is $\mathrm{O}\left(10^{-4}\right)$ s. If the shear rate is between 10 and $100 \mathrm{~s}^{-1}$, the characteristic flow time will be between $0.01 \sim 0.1 \mathrm{~s}$. Clearly, only when these two time scales are comparable we need to consider the time evolution of the bond density for computing the adhesion force. If this is not the case, which happens for the parameter values selected in this paper, it is appropriate to assume in the fluid dynamics problem that the bond density is equal to the equilibrium value for that particular bond length. The adhesion force, in this case, depends only on the distance between the cell surface and the wall, which is also the case for the potential model. The force range for the kinetic model is however much shorter.

\subsection{GOVERNING EQUATIONS}

Let us denote the domain containing the plasma and the leukocyte by $\Omega$, and the domain boundary by $\Gamma$. The plasma is modeled as a Newtonian liquid while the nucleus is modeled as a rigid sphere $\mathrm{P}(\mathrm{t})$ or as a viscoelastic liquid. The governing equations for this system are

$\nabla \cdot \mathbf{u}=0$ 


$$
\begin{gathered}
\rho\left[\frac{\partial \mathbf{u}}{\partial \mathrm{t}}+\mathbf{u} \cdot \nabla \mathbf{u}\right]=\rho \mathbf{g}-\nabla \mathrm{p}+\nabla \cdot\left(\frac{\mathrm{c}}{\lambda_{\mathrm{r}}} \mathbf{A}\right)+\nabla \cdot\left(2 \eta_{\mathrm{s}} \mathbf{D}\right)+\gamma \kappa \delta(\phi) \mathbf{n}+\mathbf{F}_{b} \delta(\phi) \\
\mathbf{u}=\mathbf{u}_{\mathrm{L}} \text { on } \Gamma, \quad \mathbf{u}=\mathbf{U}+\boldsymbol{\omega} \times \mathbf{r} \quad \text { on } \partial \mathrm{P}(\mathrm{t})
\end{gathered}
$$

The nucleus in the compound-drop model of leukocytes is assumed to be a viscoelastic liquid modeled by the Oldroyd-B model. The viscoelastic stresses, in terms of the configuration tensor $\mathbf{A}$, is given by

$$
\frac{\partial \mathbf{A}}{\partial \mathrm{t}}+\mathbf{u} \cdot \nabla \mathbf{A}=\mathbf{A} \cdot \nabla \mathbf{u}+\nabla \mathbf{u}^{\mathrm{T}} \cdot \mathbf{A}-\frac{1}{\lambda_{\mathrm{r}}}(\mathbf{A}-\mathbf{I})
$$

Here $\mathbf{u}$ is the velocity, $\mathrm{p}$ is the pressure, $\eta_{\mathrm{s}}$ is the solvent viscosity, $\rho$ is the density, $\mathbf{D}$ is the symmetric part of the velocity gradient tensor, $c$ is the polymer concentration parameter, $\lambda_{\mathrm{r}}$ is the relaxation time of the viscoelastic fluid, $\mathbf{n}$ is the outer normal, $\gamma$ is the surface tension, $\kappa$ is the surface curvature, $\phi$ is the level set function which is defined to be the distance from the interface and $\delta$ is the delta function. The polymer contribution to the viscosity is $\eta_{\mathrm{p}}=c \eta_{\mathrm{s}}$, and the zero shear viscosity of the fluid is $\eta_{\mathrm{o}}=\eta_{\mathrm{s}}+\eta_{\mathrm{p}}$.

The equations for the fluid and leukocyte motions are nondimensionalized by taking the characteristic length, velocity, time and stress scales as $R, U, R / U$ and $\eta_{0} U / R$, respectively. Here $R$ is the leukocyte radius, $\eta_{0}$ is the plasma viscosity, and $U$ is the centerline velocity of the undisturbed pressure driven flow. The nondimensional parameters are: Reynolds number $\operatorname{Re}=\rho_{0} U R / \eta_{0}$, Deborah number $D e=\mathrm{U} \lambda_{\mathrm{r}} / a$, Capillary number $\mathrm{Ca}=U \eta_{0} / \gamma$, Weber number $W e=\rho_{0} U^{2} R / \gamma$, viscosity ratios $\lambda_{1}=\eta_{0} / \eta_{1}, \lambda_{2}=\eta_{0} / \eta_{2}$, Adhesion number $A d h=F_{b} / \rho_{0} U^{2}$, and dimensionless viscosity $\eta_{s}^{\prime}=\eta_{s} / \eta_{0}$

A code based on the finite element method [22-27] is used for solving the deformation of a leukocyte in a pressure driven flow. Here, the governing equations are solved simultaneously both inside and outside the leukocyte. The cell interface is tracked using the level set method [28,22] and the adhesion force between the wall and the leukocyte is treated as a body force.

\section{RESULTS}

We next describe the direct simulation results for the motion of leukocytes in pressure driven flows in three dimensional channels with rectangular cross sections. The distance $h$ between the left and right walls of the channel is $50 \mu \mathrm{m}$, the width of the channel is $37 \mu \mathrm{m}$, and the radius of the leukocytes is 6.5 $\mu \mathrm{m}$ [5-7]. All lengths reported in this paper have been nondimensionalized with respect to the leukocyte radius. For most cases reported, the dimensionless parameters are $\mathrm{Re}=0.5, \mathrm{Ca}=0.167, \mathrm{De}=0.1$ and $\mathrm{Adh}=50.0$. For some cases $\mathrm{Re}, \mathrm{Ca}$ and Adh are varied.

\subsection{Leukocyte motion in the absence of adhesion forces.}

We first consider the case where the adhesion force between the wall and the leukocytes is not present, and thus the behavior of a leukocyte is similar to that of a drop in a pressure driven flow. In a pressure driven flow, since the shear stress is maximum at the channel wall and decreases linearly with increasing distance from the wall, the deformation is expected to be maximum when the leukocyte is close to the wall and to decrease with increasing distance from the wall. The stretching occurs at an angle of $45^{\circ}$ with the flow direction, and the stretched material elements rotate due to the flow vorticity.

Compound-drop model. Figure 4 shows the steady state leukocyte shapes for the compound-drop model at various distances from the channel wall. In all cases, both the outer layer and the nucleus of the leukocyte are deformed. Also, as noted above, the deformation of the leukocyte increases, and the angle between the principal axis of the deformed shape and the channel wall decreases, as the cell gets closer to the channel wall. In addition, the angle between the principal axis of the deformed nucleus and the wall is 
greater than that between the deformed cell and the wall. Moreover, the viscoelastic stresses inside the nucleus are maximal in the region which is closest to the wall.

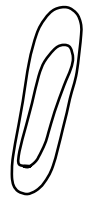

(a)

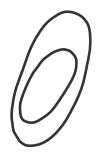

(b)

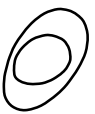

(c)

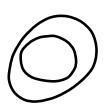

(d)

Figure 4. Leukocyte deformation in the case of the compound-drop model at different distances from the wall. The shape on the xz-mid plane through the leukocyte center are shown. (a) $\mathrm{x}=1.9$, (b) $\mathrm{x}=2.425$, (c) $\mathrm{x}=2.9$, and (d) $\mathrm{x}=3.375$ at time $\mathrm{t}=4.6$. Notice that both the leukocyte and the nucleus are deformed. The Reynolds number is 0.5 .

Drop-rigid-particle model. In the drop-rigid-particle model, the nucleus (a rigid sphere) remains spherical while the outer layer of the leukocyte deforms. The steady deformed shapes for the drop-rigid-particle model at various distances from the wall are shown in Figure 5. Figures 4 and 5 show that the overall deformation is smaller for the drop-rigid-particle model. This implies that the drop-rigid-particle model is not appropriate for situations in which the cell, as well as the nucleus, deform significantly, such as during spreading or transmigration.

Figure 6 displays the steady shape of a leukocyte released at a distance of $\mathrm{x}=2$ from the wall. It is stretched and its principal direction makes an angle of $\sim 40$ degrees with the channel wall. Our simulations show that the leukocyte is moving laterally, away from the channel wall, while moving in the flow direction. This is also the case for the compound-drop model described in the previous subsection.
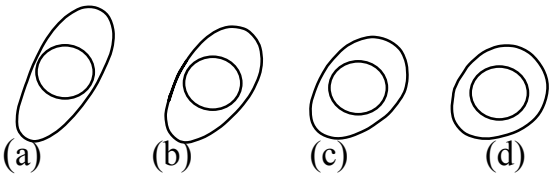

Figure 5. Leukocyte deformation in the case of the drop-rigid-particle model versus the distance from the wall. (a) $\mathrm{x}=1.9$, (b) $\mathrm{x}=2.425$, (c) $\mathrm{x}=2.9$, and (d) $\mathrm{x}=3.375$ at time $\mathrm{t}=4.6$. The deformation increases with decreasing gap between the leukocyte and the wall. Since the nucleus is rigid, it does not deform. The Reynolds number is 0.5.
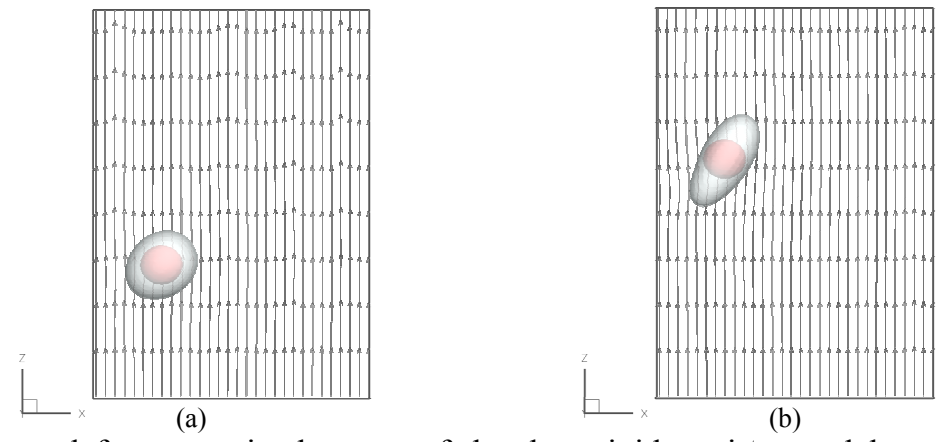

Figure 6. Leukocyte deformation in the case of the drop-rigid-particie model at (a) $t=0.82$ and (b) $t=5.25$. The Reynolds number is 0.5 .

Lateral migration of leukocytes. Past studies of the motion of drops in the pressure driven flows have shown that a drop not only moves in the flow direction, but also drifts laterally $[29,30]$. The velocity of the lateral drift, however, is very small compared to the velocity in the flow direction. Therefore, regardless of the initial position of the drop, it eventually, after a relatively large displacement in the flow direction, reaches a lateral equilibrium position, which depends on the ratio of the viscosity of the fluid inside the drop and that of the surrounding fluid.

For the parameter values considered in this study, the Reynolds number is 0.5 , and the ratio of the cytoplasm and plasma viscosities is 100. The lateral migration of leukocytes, released at different distances from the wall of the channel, is studied in a periodic domain. The initial positions ranged 
between $x=1.475$ and $x=3.375$. The channel wall is at $x=0$ and the centerline of the channel is at $x=3.85$. Some of the numerically obtained trajectories of leukocytes' centers of mass are displayed in figure 7. Our simulations show that the lateral equilibrium position of a leukocyte is around $\mathrm{x}=2.5$, which is approximately at a distance of $0.32 \mathrm{~h}$ from the channel wall, where $\mathrm{h}$ is the channel height. This implies that a leukocyte cannot be captured at a wall unless it is brought closer to the wall by some other mechanism, e.g., by collisions with other blood cells.

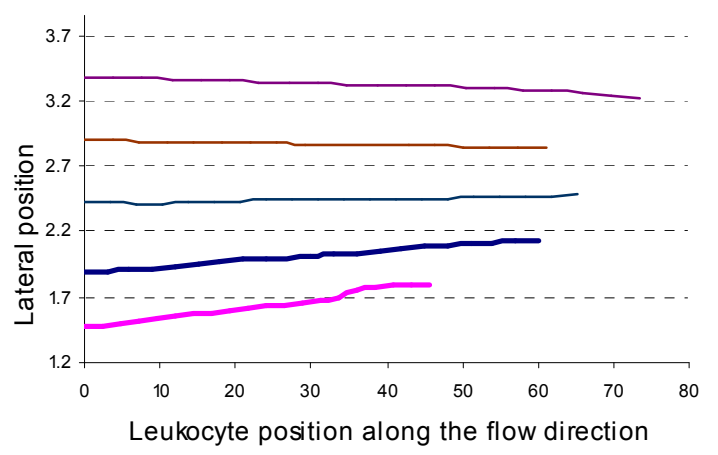

Figure 7. Trajectories of leukocytes released from different lateral positions within the channel. The Reynolds number is 0.5 .

\subsection{Leukocyte motion in the presence of adhesion forces.}

We next describe the motion of a leukocyte released near the channel wall at a distance such that the adhesion force is present. Our objective here is to study its motion and deformation under the combined action of the adhesive and hydrodynamic forces. We consider both models of the adhesive force, i.e., the potential model (1) and the kinetic model described in section 2.4.

In the presence of a pressure driven flow, the leukocyte experiences a hydrodynamic lift force whose magnitude depends on many factors including the flow velocity. If the lift force is larger than the adhesion force, the leukocyte moves away from the wall. Obviously, when the flow velocity is smaller than a critical value such that the lift force is smaller than the adhesive force, the leukocyte is expected to remain attached to the wall.

Adhesion potential model. In a pressure driven flow, in addition to the adhesion force the leukocyte also experiences a hydrodynamic force. The component of the hydrodynamic force in the flow direction, or drag, moves it in the flow direction and the component normal to the wall, or lift, moves it away from the wall, thus countering the adhesion force acting towards the wall. The adhesion potential strength and the initial leukocyte position of $x=2.0$ were selected so that the leukocyte is attracted to the wall. We first consider the case of the drop-rigid-particle model.

The flow causes the leukocyte to stretch along the shear direction, while the adhesive force pulls it towards the wall (see Figure 8). The adhesive force in the potential model is long-ranged, which can be seen by the fact that a leukocyte located at a short distance from the wall is pulled towards the wall. This feature of the potential model is perhaps unrealistic since the adhesive forces are not long-ranged. We remind the reader that the force for the kinetic model is very short-ranged. As the trailing end of the deformed leukocyte is closer to the wall, it experiences a larger attractive potential force, and thus is pulled further towards the wall. Consequently, the trailing end of the leukocyte begins to flatten, and the contact area between the leukocyte and the wall starts increasing. The contact area continues to increase until the surface tension force becomes equal to the deforming forces.

For the compound-drop model, the nucleus of the leukocyte also deforms (Figure 9). The other features of the overall deformation are similar. Here, the initial position of the center of the leukocyte is $\mathrm{x}=0.5$. The flow causes the leukocyte to move upwards, and the adhesive and shear forces cause the surface of the leukocyte near the wall to flatten. Furthermore, it is interesting to note that the gap between the leukocyte surface and the wall near the leading edge is larger than that near the trailing edge. Notice 
that this flattening of the leukocyte cannot be seen in the top view where it appears to be only elongated $[7,12]$. This is an important point because in experiments we generally have access only to the top view.

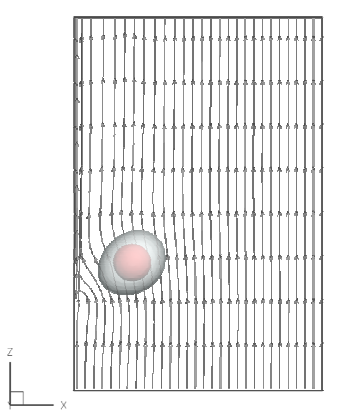

(a)

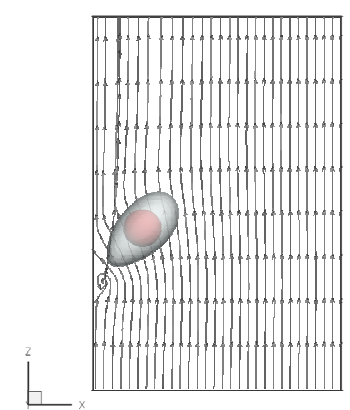

(b)

Figure 8. Deformation of a leukocyte in the case of the drop-rigid-particle model due to shear forces while it moves towards the wall under the action of the adhesive force at (a) $t=0.82$ and (b) $t=2.46$. The Reynolds number is 0.5 , $\mathrm{Ca}=0.167, \mathrm{De}=0.1$ and $\mathrm{Adh}=50.0$.

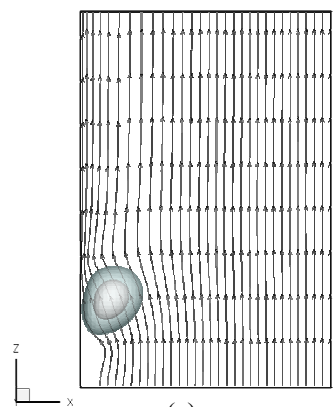

(a)

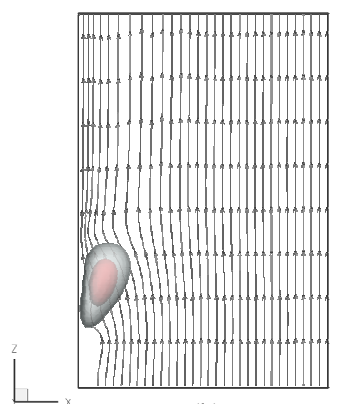

(b)

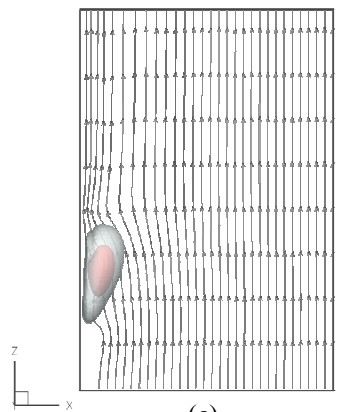

(c)

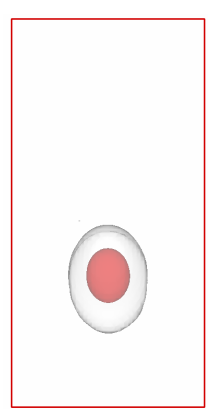

Figure 9. Leukocyte deformation for the compound-drop model released close to the wall at (a) $\mathrm{t}=0.82$, and (b) $\mathrm{t}=2.46$, and (c) $\mathrm{t}=3.94$. The top view is also shown. The Reynolds number is $0.5, \mathrm{Ca}=0.167, \mathrm{De}=0.1$ and $\mathrm{Adh}=50.0$.

Kinetic adhesion model. As noted above, the adhesion force for the kinetic model is non-zero for a very small range of distances between the leukocyte surface and the wall. Therefore, for the results presented in this section the initial position of the leukocyte is selected so that the force is present. Figure 10 shows that at the beginning the leukocyte rolls near the wall surface. The Reynolds number for this case is 0.5 . As the leukocyte deforms, the lift force it experiences increases and in this case the leukocyte moves away from the wall as the adhesion force is not sufficiently large to keep it attached. After the leukocyte moves out of the range of the kinetic adhesion force, which as noted above corresponds to the relatively short distance of about $30 \mathrm{~nm}$, the adhesion force becomes zero. The leukocyte then migrates to a lateral distance at which the lateral hydrodynamic force is zero, as discussed above.

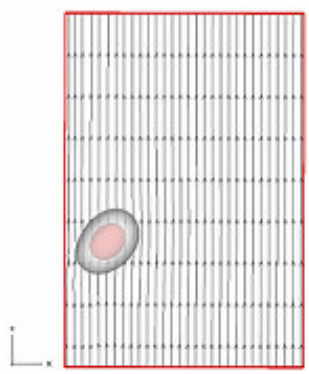

(a)

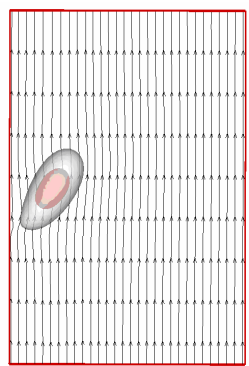

(b)

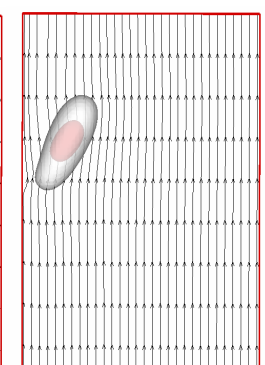

(c)

Figure 10. Leukocyte deformation in the case of the compound-drop model and the adhesion force given by the kinetic model at (a) $\mathrm{t}=0.6$, (b) $\mathrm{t}=3$, and (c) $\mathrm{t}=6$, for a Reynolds number of $\mathrm{Re}=0.5, \mathrm{Ca}=0.167, \mathrm{De}=0.1$ and $\mathrm{Adh}=50.0$. 


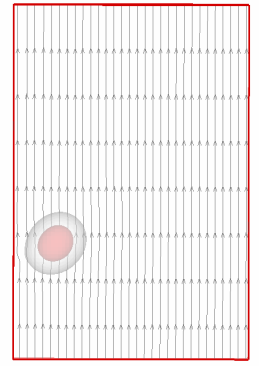

(a)

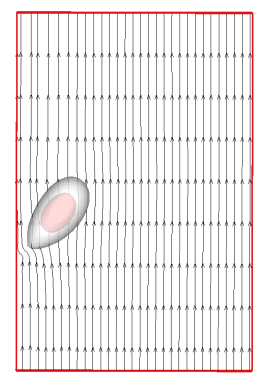

(b)

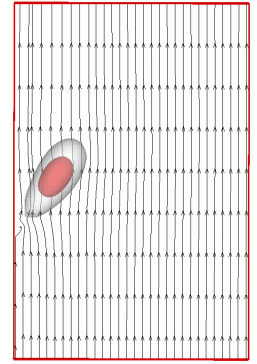

(c)

Figure 11. Leukocyte deformation in the case of the compound-drop model and the adhesion force given by the kinetic model. The parameters are selected so that the adhesion force is 100 times larger than for the case shown in Figure 10 at (a) $t=0.6$, (b) $t=3$ and (c) $t=6$. The parameters are the same as in figure 10 , except $A d h=5000.0$.

In order to ensure that the leukocyte is captured at the wall, we next consider the case where the adhesion force is 100 times larger and the adhesion parameter is 5000. In this case, the cell remained attached to the wall (Figure 11). After the steady state is reached and the deformation no longer increases, the lower area of the cell is flattened, and the leukocyte rolls and slides on the adhesive wall. In addition, the front edge of the leukocyte is farther away from the wall and the trailing end (located near the wall) is flattened. The flattening increases the surface area over which the adhesion force acts, which is especially critical in the kinetic model, as the distance range over which the force acts is rather small $[12,31]$.

\subsection{DISCUSSION AND CONCLUSIONS}

We have performed three-dimensional direct numerical simulations of the motion and deformation of a leukocyte in channels. The surface of the leukocyte is tracked using the level set method and in the case of the solid nucleus model the rigid motion enforcement inside the nucleus is performed by using the distributed Lagrange multiplier method. The leukocyte is represented using two different models: the compound-drop model and the drop-rigid-particle model. Preliminary results using this approach were presented in [32].

Our simulations show that the leukocyte deformation is larger when it is closer to the wall, where the shear rate is larger. The deformation is smaller for the drop-rigid particle model of the leukocyte. For the parameter values selected, which are typical for such flows, the leukocyte moved away from the wall to a stable location positioned between the center of the channel and the wall. This implies that the adhesion force is necessary for the leukocyte to remain attached to the wall.

In the presence of a pressure driven flow, the leukocyte experienced not only a lift force whose magnitude depends on many parameters including the flow velocity, but also a hydrodynamic torque. Both cause the trailing end of the leukocyte to move away from the wall. Clearly, when the bulk flow velocity is sufficiently small the combined effect of these hydrodynamic forces is smaller than the adhesive force, and the leukocyte remained attached to the wall. However, when the flow velocity was larger and closer to typical values, the hydrodynamic forces overcame the adhesive force and the leukocyte moved away from the wall. Our simulation results showed that for the kinetic model parameter values selected in section 2.4 and typical flow rates, the leukocyte was found to move away from the wall. This is somewhat problematic since in experiments leukocytes do get attached to the endothelium monolayer.

For the two adhesion models used in this study the trailing end of the leukocyte flattened. The flattening increases the contact surface area over which the adhesion force acts, and thus the adhesion force acting on the leukocyte. The flattening, therefore, plays an important role in increasing the adhesion force so that the latter does not get canceled by the hydrodynamic lift force and torque. The flattening of the leukocyte is particularly crucial for the kinetic model since the distance range over which the corresponding adhesive force acts is approximately $30 \mathrm{~nm}$. 


\subsection{REFERENCES}

1. Burdick, M.M., McCaffery, J.M., Kim, Y.S., Bochner, B.S., Konstantopoulos, K.,. Colon carcinoma cell glycolipids, integrins, and other glycoproteins mediate adhesion to HUVECs under flow. American Journal of Physiology Cell Physiology 284, 977-987, 2003

2. Tözeren, A., Kleinman, H.K., Grant, D.S., Morales, A.M., Byers, S.W., E-selectin-mediated dynamic of breastand colon cancer-cells with endothelial- International Journal of Cancer 60, 426-431, 1995

3. Haier, J., Nicolson, G. L.. Tumor cell adhesion under hydrodynamic conditions of fluid flow. APMIS 109, 241262, 2001

4. Chotard-Ghodsnia R., Haddad O., Leyrat A., Drochon A., Verdier C., and Duperray A., Morphological analysis of tumor cell/endothelial cell interactions under shear flow, J. Biomechanics, in press, 2006

5. Kan H.-C., Udaykumar H.S., Shyy, W., Tran-Son-Tay, R. Hydrodynamics of a compound drop with application to leukocyte modeling. Physics of Fluids 10(4), 760-774, 1998

6. N'Dri N. A., Shyy W., Tran-Son Tay R.. Computational modeling of cell adhesion and movement using a continuum-kinetics approach. Biophysical Journal 85, 2273-2286, 2003

7. Khismatullin D.B., Truskey G. A. Three-dimensional numerical simulation of a receptor-mediated leukocyte adhesion to surface: Effects of cell deformability and viscoelasticity. Physics of Fluids 17(3), 53-73, 2005

8. Bell, G.I., Dembo, M., Bongrand, P. Cell adhesion. Competition between nonspecific repulsion and specific bonding. Biophysical Journal 45(6), 1051-1064, 1984

9. Dembo M., Torney D. C., Saxman K., Hammer D. The reaction-limited kinetics of membrane-to-surface adhesion and detachment, Proceedings of the Royal Society of London - B. Biological Sciences 234(1274), 5583,1988

10. Lauffenburger D. A., Linderman J. J.. Receptors: Models for binding, trafficking, and signaling. Oxford University Press, New York. 1993

11. Sukumaran S. and Seifert U. Influence of shear flow on vesicles near a wall, Phys. Rev. E. 64, 011916, 2001

12. Khismatullin, D.B., Truskey, G.A., A 3D numerical study of the effect of channel height on leukocyte deformation and adhesion in parallel-plate flow chambers, Microvascular Research 68 (3), pp. 188-202 2004

13. Needham D. and Hochmuth R.M. Rapid flow of passive neutrophils into a $4 \mu \mathrm{m}$ pipet and measurement of cytoplasmic viscosity. Journal of Biomechanical Engineering 112 (3), 269-276, 1990

14. Schmid-Schönbein H., et al. Blood rheology and physiology of microcirculation. Ricerca in Clinica e in Laboratorio 11 Suppl 1, 13-33, 1981

15. Marc Herant, Volkmar Heinrich and Micah Dembo, Mechanics of neutrophil phagocytosis: behavior of the cortical tension. Journal of Cell Science 118 (9), 1789-1797, 2005

16. Tsai, M. A., Frank, R.S. and Waugh, R.E. Passive mechanical behavior of human neutrophils: Power-law fluid, Biophys J. 65, 2078-2088, 1993

17. Drury J.L. and Dembo M. Hydrodynamics of micropipette aspiration. Biophysical Journal 76(1), 110-128, 1999

18. Tran-Son-Tay R., Needham, D., Yeung, A., Hochmuth, R.M. Time-dependent recovery of passive neutrophils after large deformation. Biophysical Journal 60(4), 856-866, 1991

19. Bongrand, P. and Bell, G.I. Cell Surface Dynamics: Concepts and Models, Perelson, A. S., DeLisi, C., and Wiegel, F., eds., Marcel Dekker, Inc. New York, 459-493, 1984

20. Hammer, D.A., Apte, S.M., Simulation of cell rolling and adhesion on surfaces in shear flow: General results and analysis of selectin-mediated neutrophil adhesion, Biophysical Journal 63 (1), pp. 35-57,1992

21. Jin-Yu Shao, Hie Ping Ting-Beall, and Robert M. Hochmuth, Static and dynamic lengths of neutrophil microvilli, Proc. Natl. Acad. Sci. USA 95, pp. 6797-6802, 1998

22. Pillapakkam S.B., Singh P., A Level-Set Method for Computing Solutions to Viscoelastic Two-Phase Flow, Journal of Computational Physics 174, 552-578, 2001

23. Singh P. and Leal L.G., Finite element simulation of the start-up problem for a viscoelastic problem in an eccentric cylinder geometry using third-order upwind scheme, Theoretical and Computational Fluid Dynamics 5, 107-137, 1993

24. Glowinski, R., Pan, T.W., Hesla, T.I and Joseph, D.D. A distributed Lagrange multiplier/fictitious domain method for particulate flows. Int. J. of Multiphase Flows. 25 (1998), 201-233.

25. Singh P., Joseph D.D., Hesla T.I., Glowinski R.,Pan T.W., Direct numerical simulation of viscoelastic particulate flows, J. of Non Newtonian Fluid Mechanics 91, 165-188, 2000

26. Singh P. and Joseph D.D., Fluid dynamics of Floating particles, J. of Fluid Mech., 530, 31-80, 2005.

27. Kadaksham J., Singh P., Aubry N., Dynamics of pressure driven flows of electrorheological suspensions subjected to spatially non-uniform electric fields, Journal of fluids engineering 126, 170-179, 2004 
28. Sussman, M., Semereka, P. \& Osher, S., "A level set approach for computing solutions to incompressible twophase flow," J. Comput. Phys. 114, 146, 1994.

29. Segré G., Silberberg A., Behavior of macroscopic rigid spheres in Poiseuille flow. Part 2. Experimental results and interpretation, J. Fluid Mech. 14, 136-157, 1962

30. Goldsmith, H. L., Marlow J.C. The flow of model particles and blood cells and its relation to thrombogenesis. Progress in hemostasis and thrombosis 1, 97-127, 1972

31. Cantat I., and Misbah C., Dynamics and similarity laws of adhering vesicles in haptotaxis, Phys. Rev. Lett. 83, 235-238, 1999

32. Q. Jin, Verdier, C., Singh, P., Aubry, N. and Duperray, A. Direct simulation of the migration of leucocytes in pressure driven flow, ASME Paper Number FEDSM2006-98415, New York: American Society of Mechanical Engineers, 2006. 